\title{
Recombinant virus expressing hIFN- $\lambda 1$ (rL-hIFN- $\lambda 1$ ) has important effects on endoplasmic reticulum stress, autophagy and apoptosis in small cell lung cancer
}

\author{
Yulan Yan ${ }^{1 \#}$, Xiaomei Shao ${ }^{2 \#}$, Wenlu Gu ${ }^{2 \#}$, Anwei Zhang ${ }^{2}$, Xuefeng Bu ${ }^{3}$, Bing Liang ${ }^{4}$ \\ ${ }^{1}$ Department of Respiratory Medicine, Affiliated People's Hospital of Jiangsu University, Zhenjiang, China; ${ }^{2}$ Clinical Medicine College of Jiangsu \\ University, Zhenjiang, China; ${ }^{3}$ Department of General Surgery, Affiliated People's Hospital of Jiangsu University, Zhenjiang, China; ${ }^{4}$ Department of \\ Respiratory Medicine, Kunshan Hospital of Traditional Chinese Medicine, Kunshan, China \\ Contributions: (I) Conception and design: All authors; (II) Administrative support: X Bu, B Liang; (III) Provision of study materials: Y Yan, X Bu; (IV) \\ Collection and assembly of data: X Shao, W Gu; (V) Data analysis and interpretation: X Shao, W Gu, A Zhang; (VI) Manuscript writing: All authors; \\ (VII)Final approval of manuscript: All authors. \\ "These authors contributed equally to this work. \\ Correspondence to: Xuefeng Bu, PhD. Department of General Surgery, Affiliated People's Hospital of Jiangsu University, DianLi Road NO.8, \\ Zhenjiang 212002, China. Email: xuefengbu05@163.com; Prof. Bing Liang, Department of Respiratory Medicine, Kunshan Hospital of Traditional \\ Chinese Medicine, No.189 Chaoyang Road, Kunshan 215300, China. Email: liangbing3456@163.com.
}

\begin{abstract}
Background: Small cell lung cancer (SCLC) is an aggressive tumor with a poor prognosis. Human IFN- $\lambda 1$ (IL-29), belonging to the type III IFN family, captured increasing attention recently due to its crucial role in developing tumors. Recent studies have revealed that the recombinant Newcastle Disease Virus (NDV) expressing human IFN- $\lambda 1$ (rL-hIFN- $\lambda 1$ ) plays a critical role in the development of tumors. However, the role of $\mathrm{rL}-\mathrm{hIFN}-\lambda 1$ in SCLC is still unknown.

Methods: We determined the concentration of the virus intervention, followed by successfully infection in virus. We also investigated the effects of $\mathrm{rL}-\mathrm{hIFN}-\lambda 1$ on endoplasmic reticulum stress (ERS), apoptosis and autophagy in $\mathrm{H} 446$ cells, and explored the interaction among the three.

Results: We found that the ERS, autophagy and apoptosis related proteins were significantly upregulated after infected with rL-hIFN- $\lambda 1$ or NDV. In addition, both 4-phenylbutyric acid (4-PBA) or 3-Methyladenine (3-MA) could downregulate the expression of related proteins which increased by rL-hIFN- $\lambda 1$. Furthermore, we found that both B-cell lymphoma-2 (BCL-2) knockdown or Rapamycin (Rapa) could increase ERS, autophagy and apoptosis.

Conclusions: Our findings suggest that rL-hIFN- $\lambda 1$ can induce ERS, autophagy and apoptosis in SCLC H446 cells, particularly, autophagy plays an important role during this process. Furthermore, rL-hIFN- $\lambda 1$ might provide a potential biological treatment target for lung cancer treatment.
\end{abstract}

Keywords: Autophagy; endoplasmic reticulum stress (ERS); IFN- $\lambda 1$; recombinant Newcastle disease virus (NDV); small cell lung cancer (SCLC)

Submitted Feb 25, 2020. Accepted for publication Jul 18, 2020.

doi: $10.21037 /$ tcr-20-1205

View this article at: http://dx.doi.org/10.21037/tcr-20-1205

\section{Introduction}

Lung cancer is the leading cause of morbidity and mortality in men, and it is the third most common in women (1). Small cell lung cancer (SCLC) is an aggressive neuroendocrine neoplasia, characterized by rapid growth and overall poor survival (2). Despite the aggressive treatment methods have been adopted in the past decades, including radiotherapy, chemotherapy and surgery, the 5 -year survival rate of 
SCLC patients remains out of expectation. Therefore, it is necessary to develop more novel approaches to improve clinical outcomes.

One of the oncolytic virus therapy, Newcastle Disease Virus (NDV), is a new method for treating tumors $(3,4)$ as NDV can significantly inhibit the growth of tumor cells and have anti-tumor effects in a wide variety of cancers (5). IFN- $\lambda 1$, also called IL-29, is a cytokine discovered in recent years. Studies have reported that IFN- $\lambda 1$ has an anti-tumor effect, which can directly act on the tumor cells per se, or indirectly affect the anti-tumor immune mechanism to exert its anti-tumor effect (6-9). It has been found that IFN $\lambda$ s have an overt inhibitory effect on various tumors, including neuroendocrine tumors, esophageal cancer, colon cancer, liver cancer, lung adenocarcinoma, Burkitt's lymphoma, melanoma, T lymphoma and malignant glioma (6,10-12).

In the previous study, our team has successfully constructed rL-hIFN- $\lambda 1$ and found that it could induce apoptosis in gastric adenocarcinoma cells (13). Furthermore, we also found rL-hIFN- $\lambda 1$-induced endoplasmic reticulum stress (ERS) may play an important role in autophagy and apoptosis in human lung cancer A549 cells (14). However, the effect of rL-hIFN- $\lambda 1$ on SCLC H446 cells remains unclear.

In this study, we analyzed the expression of representative ERS, autophagy, and apoptosis associated proteins in rLhIFN- $\lambda 1$-infected $\mathrm{H} 446$ cells with or without pretreatment of ERS inhibitor, knockdown B-cell lymphoma-2 (BCL-2), autophagy inhibitor and autophagy enhancer. Our results suggested that $\mathrm{rL}-\mathrm{hIFN}-\lambda 1$ may play an important role in the regulation of ERS, autophagy and apoptosis in SCLC cells. This study is expected to explore a new biological method for the treatment of SCLC.

\section{Methods}

\section{Materials}

The NDV strain LaSota was provided by the Harbin Veterinary Research Institute (Harbin, Heilongjiang, China). The approach how to construct rL-hIFN- $\lambda 1$ was described in our previous published paper (15). The human SCLC cell line H446 (Cat. \# HTB-171, RRID: CVCL_1562) was obtained from the Chinese Academy of Sciences cell bank (Shanghai, China). The CCK8 kit and Rapamycin (Rapa) were acquired from HZBio (Nanjing, China). 4-phenylbutyric acid (4-PBA), Hoechst
33342, Triton X-100, Opti-MEM and 3-Methyladenine (3-MA) were purchased from Sigma-Aldrich (St. Louis, MO, USA). Lipofectamine 2000 was from Invitrogen (CA, USA). Antibodies against GRP78 (Cat. \# sc-13539, RRID: AB_627698), CHOP (Cat. \# sc-7351, RRID: AB_627411) and $\beta$-Tubulin (Cat. \# sc-69965, RRID: AB_1119492) antibodies were purchased from Santa Cruz Biotechnology (Santa Cruz, CA, USA). Antibodies against caspase- 3 and Rabbit monoclonal anti LC3 I/II antibodies were from ImmunoWay Biotechnology (Newark, DE, USA). HRPconjugated goat anti-rabbit (Cat. \# CW0103, RRID: AB_2814709) and HRP-conjugated goat anti-mouse (Cat. \# CW0102, RRID: AB_2814710) antibodies were acquired from CWBio (Shanghai, China). Cy3-conjugated goat anti-mouse antibodies were from KPL, Inc (Gaithersburg, MD, USA). siRNA against human BCL-2 was synthesized by GenePharma (Shanghai, China). Cell culture reagents were purchased from Gibco (Life Technologies, Carlsbad, CA, USA).

\section{Cell culture and infection}

H446 cells were cultured in Roswell Park Memorial Institute (RPMI 1640) medium supplemented with $10 \%$ fetal bovine serum (FBS; Gibco), $100 \mathrm{U} / \mathrm{mL}$ penicillin and $100 \mu \mathrm{g} / \mathrm{mL}$ streptomycin (Gibco) in an atmosphere of $37^{\circ} \mathrm{C}$ containing $5 \%$ carbon dioxide and subculture was conducted when the cells reached $80 \%$ confluence in the culture flask. H446 cells were inoculated in 6-well plates, and when the degree of cell confluence reached $50-70 \%$, they were randomly divided into the control group, NDV group and rl-hIFN- $\lambda 1$ group. Serum-free RPMI 1640 medium was used to dilute NDV and rl-hIFN- $\lambda 1$ respectively, the latter two groups were respectively added with diluted NDV and rl-hIFN- $\lambda 1$ virus liquid $2 \mathrm{~mL}$, cultured for $1 \mathrm{~h}$, and then the culture medium containing $10 \%$ fetal bovine serum was replaced for $24 \mathrm{~h}$ for further experiments.

\section{Cell viability assay}

The CCK-8 kit was used to quantify the cell viability according to the manufacturer's instructions. Briefly, H446 cells $\left(1 \times 10^{4} / 100 \mu \mathrm{L}\right)$ were seeded into 96 -well plate incubating $24 \mathrm{~h}$ in a humidified incubator at $37^{\circ} \mathrm{C}$, and different concentrations of the virus were then added to the plate for $24 \mathrm{~h}$. After the treatment, $10 \mu \mathrm{L}$ CCK- 8 solution was added to each well for $4 \mathrm{~h}$. A microplate reader was used to measure the spectrometric absorbance at $450 \mathrm{~nm}$. 


\section{Enzyme-linked immunosorbent assay (ELISA) analysis}

After the treatment with NDV or rl-hIFN- $\lambda 1$ for $24 \mathrm{~h}$, the supernatants of H446 cells were collected, and IL-29 levels in supernatants were measured using an ELISA kit.

\section{Western blot assay}

With or without the treatment of 4-PBA, siBCL-2, 3-MA, and Rapa, H446 cells were extracted in lysis buffer with complete protease inhibitor (Boster Biological Technology). Then, an equal amount of protein lysates was loaded on $10 \%$ polyacrylamide gel and underwent electrophoresis. The proteins were transferred to polyvinylidene difluoride (PVDF) membranes (Millipore, CA, USA). The membranes were blocked with TBST diluted 5\% skimmed milk powder for $1 \mathrm{~h}$ and incubated with primary antibodies against IFN- $\lambda 1$, GRP78, CHOP, cleaved-caspase-3, LC3 I/II, Beclin-1 and Bcl-2 at $4{ }^{\circ} \mathrm{C}$ overnight and then incubated with HRP-conjugated secondary antibodies at room temperature for $2 \mathrm{~h}$. Finally, The ECL-enhanced kit (Amersham Bioseiences, UK) was used for luminescence, and the Typhon 9400 scanner recorded the results.

\section{Small interfering RNA transfection}

When the H446 cells reached $50 \%$ confluence, siRNA transfection reagent was used according to the manufacturer's instructions. Cells pretreated with siBCL-2 were infected with NDV, IFN- $\lambda 1$ or phosphate-buffered saline (PBS), respectively. After $24 \mathrm{~h}$, the cells were harvested and analyzed by western blotting to verify the knockdown efficiency.

\section{Immunofluorescence analysis}

The lung cancer H446 cells were cultured in 24-well plates with $5 \times 10^{4}$ per well overnight, then they were infected with NDV or rl-hIFN- $\lambda 1$, while the PBS as a negative control. At $24 \mathrm{~h}$ post-infection, cells were fixed in $4 \%$ paraformaldehyde at room temperature for 20 minutes, then they were rinsed three times with PBS for 15 minutes each time. After cells were blocked in 5\% BSA for $1 \mathrm{~h}$, they were incubated with anti-GRP78 or anti-CHOP $(1: 100)$ at $4{ }^{\circ} \mathrm{C}$ overnight. Followed by being washed three times for 5 minutes each with PBS, cells were stained with Alexa Fluor 488 labeled secondary antibody or cy3labeled secondary antibody (1:500) for $1 \mathrm{~h}$, and washed with PBS for three times. Next, the nuclei were stained with nuclear fluorescence dye Hoechst 33342 for $5 \mathrm{~min}$, and then observed under a fluorescence microscope.

\section{Statistical analysis}

All data analyses were performed by SPSS V17.0 software. All results were expressed as mean \pm standard deviation. Using one-way analysis of variance and Student's $t$ tests to assess the significance of statistical differences. One-way analysis of variance and Student's $t$ tests were employed to do statistics with significance of $\mathrm{P}<0.05$ or $\mathrm{P}<0.01$.

\section{Results}

\section{Expression of viral proteins in infected SCLC cells}

We first determined the optimal virus concentration to infect cells. In this study, we used CCK- 8 analysis to examine cell viability. As shown in Figure $1 A$, as the dilution factor of the virus increased, the cell activity increased, and the rL-hIFN- $\lambda 1$ group had a cell viability $>80 \%$ at $10^{-5}$ and $10^{-6}$, while the NDV group was at $10^{-6}$. As the virus titer of cell viability $>80 \%$ was selected (15), NDV was diluted to $10^{-6}$. Additionally, rL-hIFN- $\lambda 1$ was diluted to $10^{-5}$ to assure the subsequent experimental working concentration.

Then, Western blot analysis was performed to monitor the expression of IFN- $\lambda 1$ in $\mathrm{H} 446$ cells. After H446 cells were infected by rL-hIFN- $\lambda 1$ and NDV, Western blot showed that the expression of IFN- $\lambda 1$ protein in $\mathrm{rL}$ hIFN- $\lambda 1$ group was significantly higher than the control group and NDV group (Figure 1B).

In addition, ELISA analysis was performed to evaluate IFN- $\lambda 1$ expression. The expression of IFN $-\lambda 1$ was significantly increased in NDV group and rL-hIFN- $\lambda 1$ group after infection with $\mathrm{rL}-\mathrm{hIFN}-\lambda 1$ and NDV. In the supernatant of different viral dilution concentrations, the expression levels of IFN- $\lambda 1$ was obviously increased in $\mathrm{rL}$ hIFN- $\lambda 1$ group compared to NDV group, and IFN- $\lambda 1$ increased dramatically while the virus was diluted 100-fold compared with 200 -fold in rL-hIFN- $\lambda 1$ group (Figure $1 C$ ).

\section{$r L-b I F N-\lambda 1$ induces ERS response, autophagy and apoptosis in $\mathrm{H} 446$ cells}

Immunofluorescence imaging was carried out to investigate whether rL-hIFN- $\lambda 1$ could induce ERS in SCLC H446 cells. As shown in Figure 2A, ERS related proteins (GRP78, CHOP) were dramatically elevated in the rL-hIFN- $\lambda 1$ 


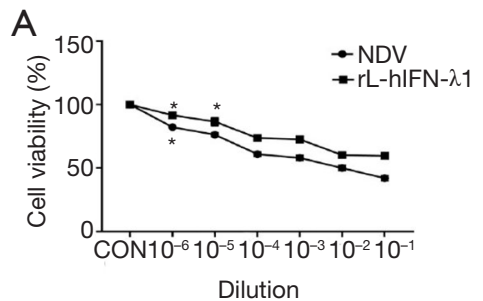

C

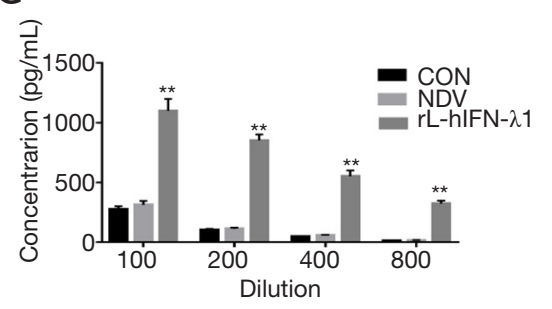

B
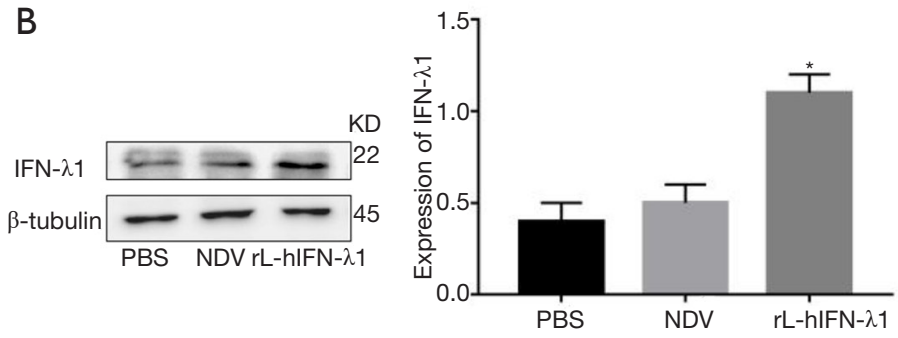

Figure 1 Expression of viral proteins in infected small cell lung cancer cells. (A) H446 cells were infected by different solubilities of rLhIFN- $\lambda 1$ or NDV and cell viability was examined by CCK-8 analysis; (B) Western blot was used to test the protein of IFN- $\lambda 1$ in $\mathrm{H} 446$ cells after infected by rL-hIFN- $\lambda 1$ or NDV; (C) H446 cells were treated with different solubilities of rL-hIFN- $\lambda 1$ or NDV and the content of IFN- $\lambda 1$ was quantified by ELISA assay. * $\mathrm{P}<0.05$; ** $\mathrm{P}<0.01$. rL-hIFN- $\lambda 1$, recombinant Newcastle Disease Virus expressing human IFN- $\lambda 1$. NDV, Newcastle Disease Virus. IFN- $\lambda 1$, recombinant Newcastle Disease Virus.

group and the NDV group compared with the PBS group. Same as this result, the expression of their related proteins (GRP78, CHOP) was assessed by Western blot analysis (Figure 2B). Then, the expression levels of ERS related proteins (GRP78, CHOP) were measured after treating with ERS inhibitor (4-PBA) with the result that the expression levels of GRP78 and CHOP were significantly decreased in 4-PBA pretreatment group (Figure 2B).

Furthermore, the Figure $2 C$ revealed that rL-hIFN- $\lambda 1$ contributed to autophagy and apoptosis in H446 cells. In detail, Western blot suggested that the expression of autophagy related protein LC3I/II and apoptosis related protein cleaved-caspase- 3 were significantly increased in the rL-hIFN- $\lambda 1$ group compared with the control group and the NDV group after H446 cells were infected by rL-hIFN- $\lambda 1$ or NDV. On the contrary, autophagy and apoptosis related proteins were significantly downregulated by treating with 4-PBA.

These results demonstrated that rL-hIFN- $\lambda 1$ could induce ERS, autophagy and apoptosis, and the inhibition of ERS could reduce autophagy and apoptosis.

\section{Knockout of Bcl-2 can promote ERS, autophagy and apoptosis in SCLC H446 cells}

Given the fact that Bcl-2 protein family has important effects on autophagy and apoptosis (16-18), we transfected H446 cells with Bcl-2 siRNA. Green fluorescent cells were observed under fluorescence microscope, indicating successful siRNA transfection (Figure 3A). Western blot showed that the protein expression of Bcl-2 was obviously reduced after siRNA treatment, illustrating that gene knockout was effective. Subsequently, we detected the expression of ERS related proteins (CHOP, GRP78), autophagy related proteins (LC3 I/II, beclin-1) and apoptosis related protein (cleaved-caspase-3). As expected, the expressions of these proteins were markedly higher than those in the untreated group (Figure 3B).

\section{Autophagy plays an important role in ERS, autophagy and apoptosis in SCLC H446 cells}

Moreover, to validate the role of autophagy in ERS and apoptosis, autophagy inhibitor (3-MA) and autophagy enhancer (Rapa) were performed in this study. After treatment with 3-MA, the expression level of LC3 I/II was significantly decreased compared with the untreated group. In addition, the expression levels of ERS related proteins (CHOP, GRP78) and apoptosis related protein (cleavedcaspase-3) were significantly reduced in comparison to the untreated group (Figure 4A). Interestingly, after treatment with Rapa, Western blot showed the opposite result 
A

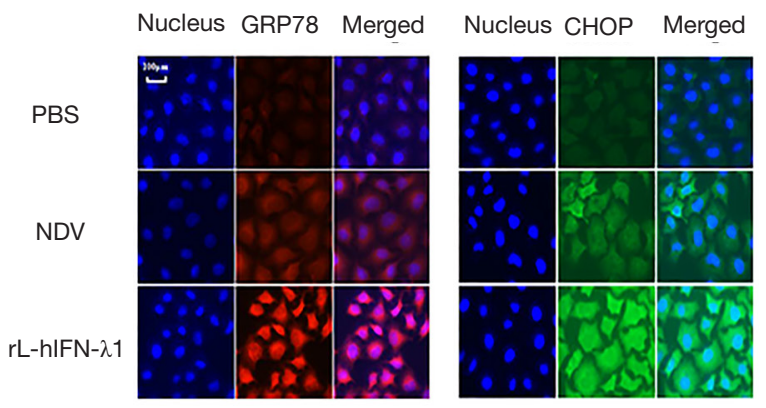

B
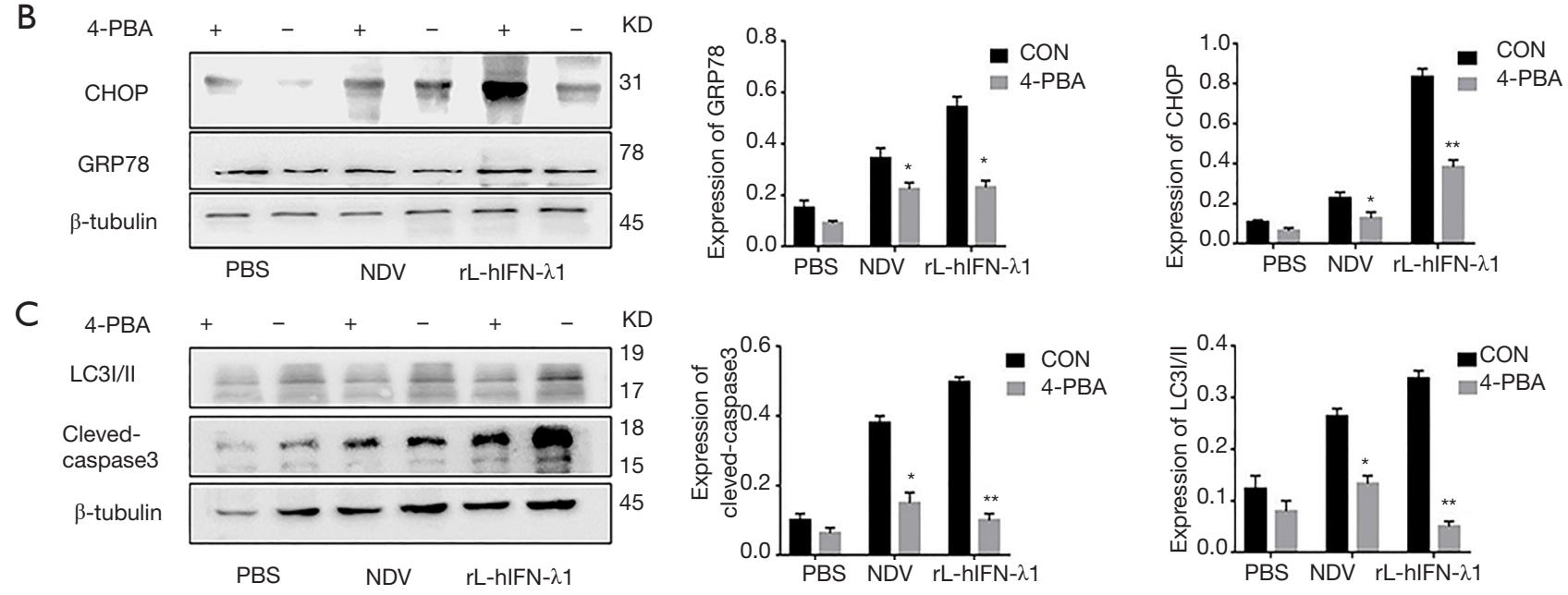

Figure 2 rL-hIFN- $\lambda 1$ induces the endoplasmic reticulum stress response, autophagy and apoptosis in H446 cells. (A) After infection of 4446 cells by rL-hIFN- $\lambda 1$ or NDV, the expression of CHOP and GRP78 proteins were detected by immunofluorescence. The red fluorescence was GRP78 protein, and the green fluorescence was CHOP protein; (B) after 4-phenylbutyric acid pretreatment, H446 cells were infected with rL-hIFN- $\lambda 1$ or NDV, and the expression of endoplasmic reticulum stress related proteins were detected by Western blot; (C) Western blot was used to test the protein of autophagy and apoptosis related proteins in H446 cells after infected by rL-hIFN- $\lambda 1$ or NDV. *, $\mathrm{P}<0.05$; ${ }^{* *}, \mathrm{P}<0.01$. rL-hIFN- $\lambda 1$, recombinant Newcastle Disease Virus expressing human IFN- $\lambda 1$. NDV, Newcastle Disease Virus.

(Figure 4B). Altogether, these results demonstrated that autophagy played an important role in ERS, autophagy and apoptosis in SCLC H446 cells.

\section{Discussion}

Lung cancer is one of the malignancies with the highest morbidity and mortality rate and the greatest threat to human's health and life. At present, the incidence of lung cancer in China is $53.57 / 100,000$, and the mortality rate in the same period is as high as $45.57 / 100,000$, ranking first in the incidence and mortality of malignant tumors in China (19). Despite the rapid development of detection and treatment, the long-term survival rate of lung cancer remains low. Therefore, we need to find a new type of treatment to improve the survival rate of lung cancer.

Oncolytic therapy is a novel biological treatment. Oncolytic viruses can destroy harmful cells by infecting and replicating in cancer cells while leaving normal cells unaffected (20). In recent decades, NDV has been shown to induce tumor cell death via its oncolytic effects (21). Previous studies have reported that NDV may upregulate TRAIL expression in NK cells through the IFN- $\gamma$ receptor pathway (22), and killer activation receptors pathway participated in the IFN- $\gamma$-independent TRAIL expression of NDV-stimulated NK cells through the activation of Syk and NF- $\kappa \mathrm{B}$ (23). The main functions of IFN- $\lambda 1$ include antiviral, anti-tumor proliferation and immunomodulatory effects $(12,24)$. Previous reports have shown that IFN- $\lambda 1$ induces autophagy and apoptosis in lung adenocarcinoma 

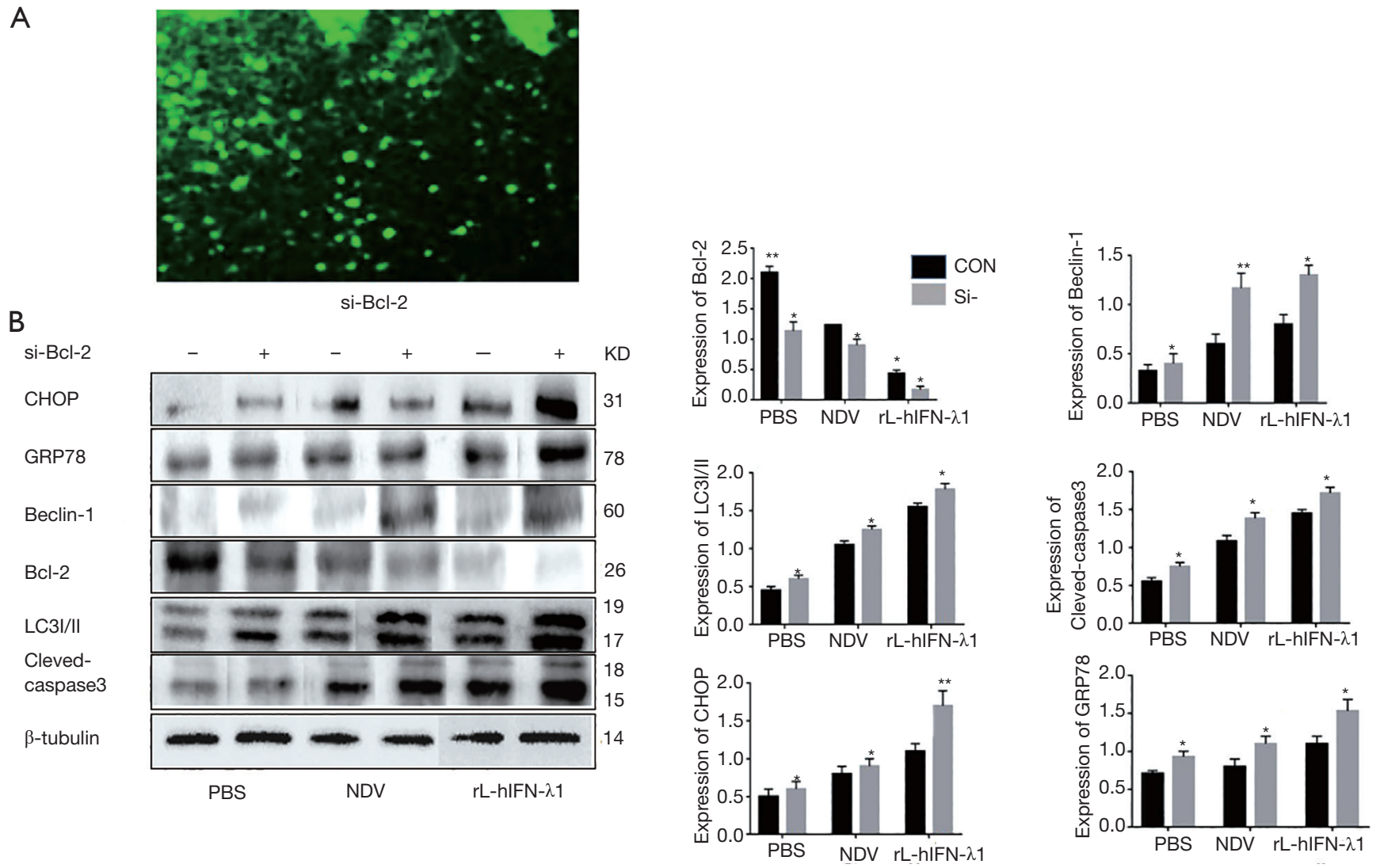

Figure 3 Knockout of BCL-2 can promote endoplasmic reticulum stress, autophagy and apoptosis in small cell lung cancer H446 cells. (A) Infection efficiency was determined after siRNA-Bcl-2 interfered with H446 cells; (B) related proteins (Bcl-2, Beclin-1, GRP78, CHOP, LC3 I/II and cleaved-caspase-3) were detected by western blot after treated with siRNA. *, P<0.05; **, P<0.01. BCL-2, B-cell lymphoma-2.

cells through ERS (14).

In this study, the effect of different concentrations of virus on the viability of tumor cells was firstly tested by CCK-8. We used a relatively safe virus concentration, NDV was selected to dilute $10^{-6}$, and rL-hIFN- $\lambda 1$ was selected to be diluted $10^{-5}$ for subsequent experiments. Then, ELISA and Western blot results showed that IFN $-\lambda 1$ could be stably expressed and proved successful infection, which also provided the basis for subsequent experiments.

Under normal conditions, endoplasmic reticulum transmembrane protein (GRP78/BIP) binds to glucoseregulated protein to inhibit the polymerization of GRP78, resulting in inactive state of GRP78. However, when ERS occurs, GRP78 is released and polymerized with transmembrane proteins, eventually triggering unfolded protein response (UPR) (25). In addition, previous studies have shown that when ERS occurs, an increase in CHOP and GRP78 indicates the occurrence of UPR (26). In the present study, we found that CHOP and GRP78 were significantly elevated after infection of NDV and rLhIFN- $\lambda 1$, indicating that $\mathrm{LL}-\mathrm{hIFN}-\lambda 1$ can induce ERS in SCLC H446 cells.

4-PBA promotes proteins transport in the endoplasmic reticulum to the cytoplasm and reduces endoplasmic reticulum load. It was therefore frequently used as an inhibitor of ERS. In our study, H446 cells were pretreated with 4-PBA. The expression of ERS related proteins GRP78 and CHOP in rL-hIFN- $\lambda 1$ group was significantly lower than in untreated group. Furthermore, after 4-PBA treatment, the expression of autophagy related protein LC3I/II and apoptosis related protein cleaved-caspase-3 in $\mathrm{rL}-\mathrm{hIFN}-\lambda 1$ group also decreased significantly. These results suggested that 4-PBA could inhibit the expression of ERS, autophagy and apoptosis related proteins induced by rL-hIFN- $\lambda 1$.

Autophagy is not only a process of self-feeding during 
A

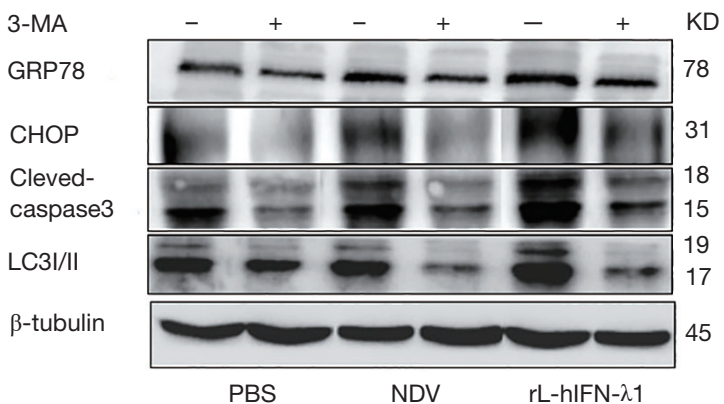

B
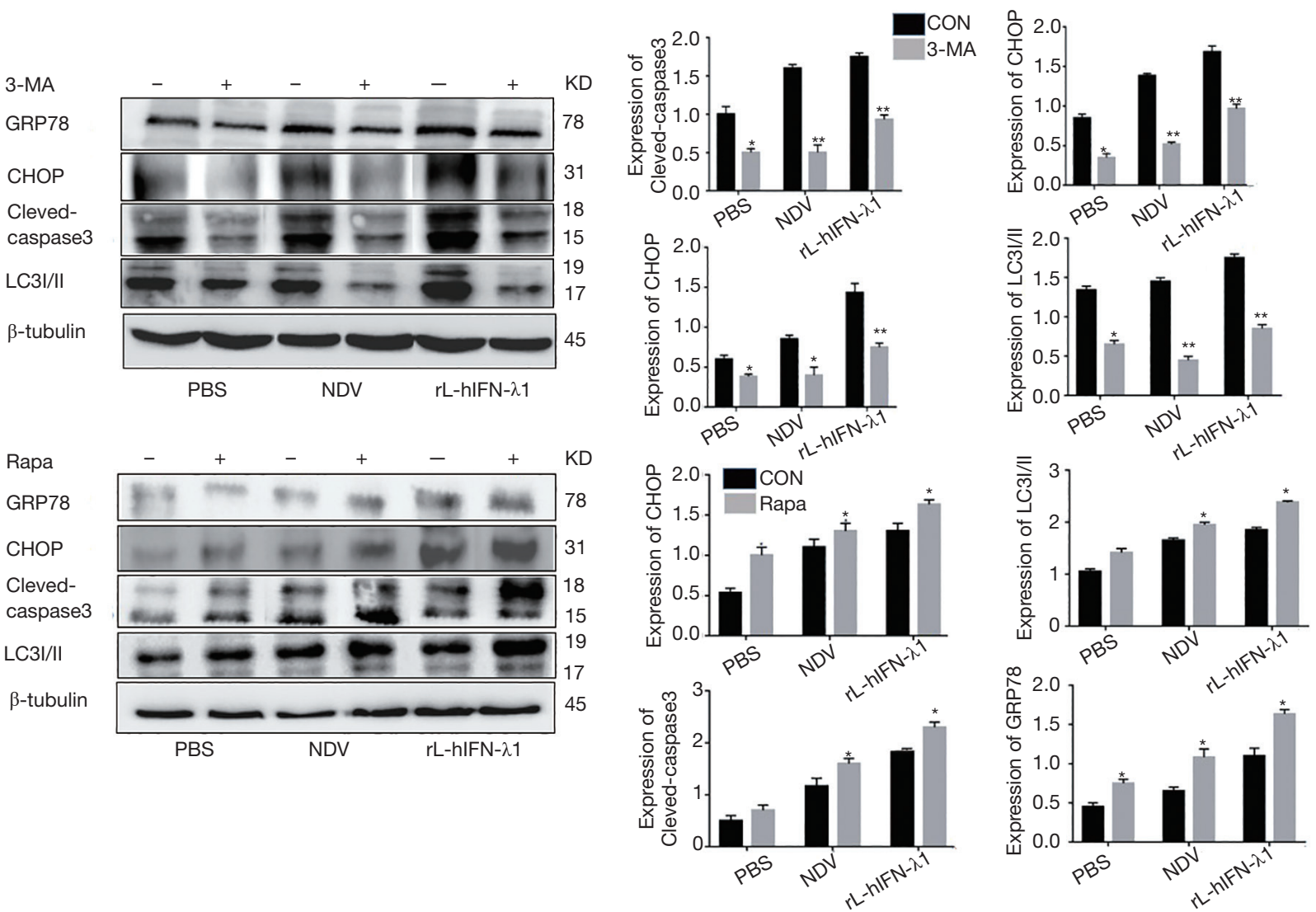

Figure 4 Autophagy plays an important role in endoplasmic reticulum stress, autophagy and apoptosis in small cell lung cancer H446 cells. (A) Western blot was used to detect the level of endoplasmic reticulum stress related proteins (CHOP, GRP78), autophagy related proteins (LC3 I/II) and apoptosis related protein (cleaved-caspase-3) after treated with 3-Methyladenine; (B) the expression of related proteins (GRP78, CHOP, LC3 I/II and cleaved-caspase-3) were significantly upregulated after treated with Rapamycin. *, $\mathrm{P}<0.05 ;$ **, $\mathrm{P}<0.01$.

hunger or stress, but also an important pathway for protein degradation. Intracellular basic autophagy plays an important role in protein damage, dysfunction, and degradation of organelles. Studies have indicated that autophagy-deficient mice have accumulated false-folded, damaged proteins (27). The medication between ERS and autophagy was regulated by three main mechanisms, involving PERK/eIF2 $\alpha$, IRE1 and intracellular Ca2 ${ }^{+}$ concentration. Inhibition of autophagy by IRE1 under ERS was achieved by directly promoting the expression of apoptosis-inhibiting protein $\mathrm{Bcl}-2$ and inhibiting the activity of Beclin1. Phosphorylation of Bcl-2 led to dissociation of Beclin-1/Bcl-2 complex and activation of autophagy occurred in the early stages of ERS $(28,29)$. Previous studies have shown that rL-hIFN- $\lambda 1$ could lead to ERS, autophagy and apoptosis, possibly through the IRE1/JNK/bcl-2 pathway (14). After knockdown of BCL-2, ERS related proteins (CHOP, GRP78), we found that autophagyassociated proteins (LC3I/II, beclin-1) and apoptosis related proteins (cleaved-caspase-3) were significantly higher than those of untreated groups. Therefore, we hypothesized that knocking out BCL-2 played an important role in promoting ERS, autophagy and apoptosis.

Previous studies have elucidated that the effects of autophagy activity on a variety of malignant tumors have two sides: on one hand, the ability of tumor cells to maintain their survival can be enhanced by upregulating the autophagy activity to increase the tolerance to metabolic stress; on the other hand, cells may die due to excessive autophagy, and autophagy reduces the stimulation of aberrated organelles to the carcinogenesis of cells mainly by phagocytizing damaged organelles or cytoplasmic proteins $(30,31)$. Therefore, we explored the effects of autophagy on SCLC H446 cells. Western blot showed that the expression 
of LC3 I/II was substantially decreased after intervention with autophagy inhibitor 3-MA. In addition, the expression of CHOP, GRP78 and cleaved-caspase- 3 were also decreased. Consistent with this, the opposite result was obtained after treatment with the autophagy enhancer Rapamycin. We proposed that autophagy was associated with ERS and apoptosis, and interacted with each other.

\section{Conclusions}

In conclusion, we mainly elucidated that rL-hIFN- $\lambda 1$ could induce ERS, autophagy and apoptosis in SCLC H446 cells, and autophagy played an important role in it, and rLhIFN- $\lambda 1$ is expected to become a biological treatment for lung cancer treatment.

\section{Acknowledgments}

We are grateful to the Key Laboratory of Veterinary Biotechnology, Harbin Veterinary Research Institute of the Chinese Academy of Agricultural Sciences for the help of rL-hIFN- $\lambda 1$. We would also thank Zhijian Zhang from Jiangsu University (Jiangsu, China) for the helpful suggestions.

Funding: This study was financially supported by the Project of Natural Science Foundation of Jiangsu Province, China (BK20151333); Key R \& D Program Project of Zhenjiang, China (SH2018059) and Social Development Project of Kunshan, China (KS1653).

\section{Footnote}

Data Sharing Statement: Available at http://dx.doi. org/10.21037/tcr-20-1205

Conflicts of Interest: All authors have completed the ICMJE uniform disclosure form (available at http://dx.doi. org/10.21037/tcr-20-1205). The authors have no conflicts of interest to declare.

Ethical Statement: The authors are accountable for all aspects of the work in ensuring that questions related to the accuracy or integrity of any part of the work are appropriately investigated and resolved.

Open Access Statement: This is an Open Access article distributed in accordance with the Creative Commons Attribution-NonCommercial-NoDerivs 4.0 International
License (CC BY-NC-ND 4.0), which permits the noncommercial replication and distribution of the article with the strict proviso that no changes or edits are made and the original work is properly cited (including links to both the formal publication through the relevant DOI and the license). See: https://creativecommons.org/licenses/by-nc-nd/4.0/.

\section{References}

1. Bray F, Ferlay J, Soerjomataram I, et al. Global cancer statistics 2018: GLOBOCAN estimates of incidence and mortality worldwide for 36 cancers in 185 countries. CA Cancer J Clin 2018;68:394-424.

2. Früh M, De Ruysscher D, Popat S, et al. Small-cell lung cancer (SCLC): ESMO Clinical Practice Guidelines for diagnosis, treatment and follow-up. Ann Oncol 2013;24 Suppl 6:vi99-105.

3. Khalighinejad N, Hariri H, Behnamfar O, et al. Adenoviral gene therapy in gastric cancer: a review. World J Gastroenterol 2008;14:180-4.

4. Janke M, Peeters B, de Leeuw O, et al. Recombinant Newcastle disease virus (NDV) with inserted gene coding for GM-CSF as a new vector for cancer immunogene therapy. Gene Ther 2007;14:1639-49.

5. Reichard KW, Lorence RM, Cascino CJ. Selective replication of Newcastle disease virus (NDV) in cancer cells is associated with virus-induced cell fusion. Proc Am Assoc Cancer Res 1992;33:521.

6. Guenterberg KD, Grignol VP, Raig ET, et al. Interleukin-29 binds to melanoma cells inducing JakSTAT signal transduction and apoptosis. Mol Cancer Ther 2010;9:510-20.

7. Sinha S, Guo Y, Thet S, et al. IFN type I and type II independent enhancement of B cell TLR7 expression by natural killer cells. J Leukoc Biol 2012;92:713-22.

8. He SH, Chen $\mathrm{X}$, Song $\mathrm{CH}$, et al. Interferon- $\lambda$ mediates oral tolerance and inhibits antigen-specific, T-helper 2 cell-mediated inflammation in mouse intestine. Gastroenterology 2011;141:249-58.e2582.

9. Hui X, Chen H, Zhang S, et al. Antitumor activities of recombinant human interferon (IFN)-lambda1 in vitro and in xenograft models in vivo for colon cancer. Cancer Lett 2011;311:141-51.

10. Witte K, Witte E, Sabat R et al. IL-28A, IL-28B, and IL-29: promising cytokines with type I interferon-like properties. Cytokine Growth Factor Rev 2010;21:237-51.

11. Li Q, Kawamura K, Ma G, et al. Interferon-lambda induces $\mathrm{G} 1$ phase arrest or apoptosis in oesophageal 
carcinoma cells and produces anti-tumour effects in combination with anti-cancer agents. Eur J Cancer 2010;46:180-90.

12. Sato A, Ohtsuki M, Hata M, et al. Antitumor activity of IFN-lambda in murine tumor models. J Immunol 2006;176:7686-94.

13. Bu X, Li M, Zhao Y, et al. Genetically engineered Newcastle disease virus expressing human interferon- $\lambda 1$ induces apoptosis in gastric adenocarcinoma cells and modulates the Th1/Th2 immune response. Oncol Rep 2016;36:1393-402.

14. Yan Y, Liu S, Li M, et al. Recombinant Newcastle disease virus expressing human IFN-lambda1 (rL-hIFNlambda1)-induced apoptosis of A549 cells is connected to endoplasmic reticulum stress pathways. Thorac Cancer 2018;9:1437-52.

15. Wang YJ, Zhou Q, Cao S, et al. Efficient gene therapy with a combination of ultrasoundtargeted microbubble destruction and PEI/DNA/NLS complexes. Mol Med Rep 2017;16:7685-91.

16. Yoo YM, Han TY, Kim HS. Melatonin Suppresses Autophagy Induced by Clinostat in Preosteoblast MC3T3-E1 Cells. Int J Mol Sci 2016;17:526.

17. Levine B, Sinha S, Kroemer G. Bcl-2 family members: dual regulators of apoptosis and autophagy. Autophagy 2008;4:600-6.

18. Zhou F, Yang Y, Xing D. Bcl-2 and Bcl-xL play important roles in the crosstalk between autophagy and apoptosis. Febs j 2011;278:403-13.

19. Siegel RL, Miller KD, Jemal A. Cancer statistics, 2016. CA Cancer J Clin 2016;66:7-30.

20. Ali R, Alabsi AM, Ali AM, et al. Cytolytic effects and apoptosis induction of Newcastle disease virus strain AF2240 on anaplastic astrocytoma brain tumor cell line. Neurochem Res 2011;36:2051-62.

21. Sinkovics JG, Horvath JC. Newcastle disease virus (NDV): brief history of its oncolytic strains. J Clin Virol
2000;16:1-15.

22. Song DZ, Liang Y, Xiao Q, et al. TRAIL is involved in the tumoricidal activity of mouse natural killer cells stimulated by Newcastle disease virus in vitro. Anat Rec (Hoboken) 2013;296:1552-60.

23. Liang Y, Song DZ, Liang S, et al. The hemagglutininneuramidinase protein of Newcastle disease virus upregulates expression of the TRAIL gene in murine natural killer cells through the activation of Syk and NFkappaB. PLoS One 2017;12:e0178746.

24. Gough L, Schulz O, Sewell HF, et al. The cysteine protease activity of the major dust mite allergen Der $\mathrm{p}$ 1 selectively enhances the immunoglobulin E antibody response. J Exp Med 1999;190:1897-902.

25. Walter P, Ron D. The unfolded protein response: from stress pathway to homeostatic regulation. Science 2011;334:1081-6.

26. Kim SR, Kim HJ, Kim DI, et al. Blockade of Interplay between IL-17A and Endoplasmic Reticulum Stress Attenuates LPS-Induced Lung Injury. Theranostics 2015;5:1343-62.

27. Kuma A, Hatano M, Matsui M, et al. The role of autophagy during the early neonatal starvation period. Nature 2004;432:1032-6.

28. Pattingre S, Tassa A, Qu X, et al. Bcl-2 antiapoptotic proteins inhibit Beclin 1-dependent autophagy. Cell 2005;122:927-39.

29. Yamamoto K, Ichijo H, Korsmeyer SJ. BCL-2 is phosphorylated and inactivated by an ASK1/Jun $\mathrm{N}$-terminal protein kinase pathway normally activated at G(2)/M. Mol Cell Biol 1999;19:8469-78.

30. Apel A, Zentgraf H, Buchler MW, et al. Autophagy-A double-edged sword in oncology. Int J Cancer 2009; 125:991-5.

31. Choi KS. Autophagy and cancer. Exp Mol Med 2012;44:109-20.
Cite this article as: Yan $\mathrm{Y}$, Shao $\mathrm{X}, \mathrm{Gu} W$, Zhang $\mathrm{A}, \mathrm{Bu}$ $X$, Liang B. Recombinant virus expressing hIFN $\lambda 1$ (rLhIFN- $\lambda 1$ ) has important effects on endoplasmic reticulum stress, autophagy and apoptosis in small cell lung cancer. Transl Cancer Res 2020;9(9):5209-5217. doi: 10.21037/tcr-20-1205 